\title{
Ecoturismo implementado en el mundo globalizado como alternativa de desarrollo económico y social
}

\section{Ecotourism implemented in the globalized world as an alternative to economic and social development}

\author{
Edwin Eduardo Millán-Rojas ${ }^{1}$, , Verenice Sánchez-Castillo $^{2}{ }^{\mathbb{D}}$ y Carlos Alberto Gómez-Cano ${ }^{3}$ (D) \\ ${ }^{1}$ Universidad de la Amazonía, Colombia.Email: e.millan@udla.edu.co \\ ${ }^{2}$ Universidad de la Amazonía, Colombia. Email: ve.sanchez@udla.edu.co \\ ${ }^{3}$ Universidad de la Amazonía, Colombia. Email: ca.gomez@udla.edu.co \\ Para citar este artículo: Millán, R. E., Sánchez, C. V. y Gómez, C. C. (2020). Ecoturismo implementado en el mundo \\ globalizado como alternativa de desarrollo económico y social. Clío América, 14(27), 380-389. \\ http://dx.doi.org/10.21676/23897848.3433
}

Recibido: 20 septiembre de 2019

Aceptado: 10 de diciembre de 2019

Publicado en línea: marzo 03 de 2020

Palabras clave: conservación ambiental; desarrollo comunitario; desarrollo sostenible; educación ambiental; economía verde. JEL: Q260; Q570; R110

\section{Keywords:} community development; environment al conservation; environment al education; green economy; sustainable development.

\section{RESUMEN}

El presente trabajo desarrolla los fundamentos teóricos del ecoturismo como alternativa de desarrollo social y económico en el mundo globalizado. Se utilizó el método exploratorio descriptivo con el propósito de indagar experiencias significativas en diferentes países, buscando los impactos positivos y negativos del ecoturismo en diferentes contextos. Se logró demostrar la necesidad de acompañar las iniciativas empresariales del ecoturismo con una educación ambiental para mitigar el gasto del recurso ambiental ocasionado por su sobreexplotación. Al final se deja evidencia de los diferentes casos de éxito y problemáticas generadas a partir del desarrollo social y económico ocasionado por el ecoturismo en diferentes comunidades en el mundo, donde se evidencia que a pesar de estar en sitios geográficos distantes, los problemas generalmente son los mismos. Por último, es necesario ampliar a nuevos trabajos en los que se sistematicen las prácticas tangibles de la educación ambiental y cómo esta impacta de manera real en el desarrollo de las comunidades.

\section{ABSTRACT}

This work develops the theoretical foundations of ecotourism as an alternative for social and economic development in the globalized world. The descriptive exploratory method was used with the purpose of investigating significant experiences in different countries, looking for the positive and negative impacts of ecotourism in different contexts. It was possible to demonstrate the need to accompany ecotourism business initiatives with an environmental education to mitigate the expense of the environmental resource caused by its overexploitation. In the end we leave evidence of the different cases of success and problems generated from the social and economic development generated by ecotourism in different communities in the world, where it is evident, despite being in distant geographical positions, the problems are generally the same. Finally, it is necessary to expand to new works where the tangible practices of environmental education are systematized and how these impacts in a real way the development of the communities. 


\section{INTRODUCCIÓN}

En este trabajo se dan a conocer las principales características del ecoturismo implementado en el mundo globalizado como alternativa de desarrollo económico y social, tomando como referente países en diferentes contextos geográficos. El trabajo está enmarcado en el proyecto de investigación "Estrategias socioculturales y ambientales para el fomento del eco-turismo comunitario en el municipio de Florencia, Caquetá", desarrollado por investigadores de la Universidad de la Amazonia y financiado por el "Patrimonio autónomo Fondo Nacional de Financiamiento para la Ciencia, la Tecnología y la Innovación Francisco José de Caldas" en convenio con Colciencias, ahora Ministerio de Ciencia y Tecnología e Innovación de Colombia. Esto debido al interés presente por reconocer e identificar cómo se debe realizar investigación desde este ámbito. Teniendo en cuenta lo anterior, se desarrolló una revisión de las diferentes estrategias generadas en algunos países relacionadas con la promoción y realización de prácticas ecoturísticas como alternativa de desarrollo económico y social.

El problema analizado está basado en la necesidad de describir referentes de éxito o fracaso en la implementación de estrategias para el fomento del ecoturismo, para lo cual se dividió el problema en subproblemas relacionados: ¿cuáles son los vacíos conceptuales presentes en el uso del ecoturismo como alternativa de desarrollo económico y social?, ¿cómo se desarrolla el ecoturismo a lo largo de los años en las comunidades?, ¿cuál es la discusión actual sobre el ecoturismo? y ¿cuáles son los temas relevantes del ecoturismo enfocado al desarrollo económico y social de las poblaciones?

A nivel internacional se encuentran múltiples referentes teóricos de experiencias significativas con impactos positivos y negativos (Lenao y Basupi, 2016; Ospina et al., 2019). A lo largo de los últimos años han tomado importancia los desarrollos turísticos como fuentes de ingreso para los países desarrollados y como una alternativa económica para países en vía de desarrollo. Sin embargo, esto también ha ocasionado la necesidad de involucrar otras áreas del conocimiento como la educación ambiental (Silva, 2017) para mitigar los diferentes impactos generados por este crecimiento en las zonas turísticas en diferentes partes del mundo.

Para el desarrollo de la presente investigación se analizaron las categorías de ecoturismo o turismo de naturaleza, ecoturismo comunitario, educación ambiental y desarrollo económico y social, como ejes teóricos para el estudio del ecoturismo implementado en el mundo globalizado como alternativa de desarrollo económico y social. En principio se definieron las categorías y las percepciones de los autores sobre el concepto y la estructura de cada categoría, para luego, a partir de estas definiciones, hacer un análisis geográfico de los casos de éxito en el uso del ecoturismo.

Se logró identificar experiencias significativas de problemas y soluciones en diferentes contextos geográficos (Zamora y Riveros, 2016), las cuales pueden ser replicadas en otros escenarios locales. Además se identificó la estrecha relación en el desarrollo de las comunidades con la educación ambiental necesaria para lograr modelos sostenibles y sustentables en la línea del tiempo y de esa manera lograr un desarrollo económico y social perdurable en las comunidades.

\section{METODOLOGÍA}

El presente estudio se efectuó desde una óptica del método exploratorio con un enfoque descriptivo, aplicando el método se hizo una búsqueda en las bases de datos de Science Direct, Web Science y Scopus acerca de las experiencias significativas relacionadas con el ecoturismo y su implementación como alternativa de desarrollo económico y social. Para realizar las búsquedas se abordaron las categorías de ecoturismo, ecoturismo comunitario, turismo de naturaleza y educación ambiental, logrando obtener un universo de 250 artículos relacionados en las diferentes categorías. Posteriormente a la búsqueda se procedió a hacer el análisis basado en las siguientes preguntas de investigación y la contribución hecha por los artículos a dar respuesta a los interrogantes planteados: ¿cuáles son los vacíos conceptuales presentes en el uso del ecoturismo como alternativa de desarrollo económico y social?, ¿cómo se desarrolla el ecoturismo a lo largo de los años en las comunidades?, ¿cuál es la discusión actual sobre el ecoturismo? y ¿cuáles son los temas relevantes del ecoturismo enfocado al desarrollo económico y social de las poblaciones? A partir de estos cuatro cuestionamientos se hizo una descripción detallada de las experiencias presentes en los trabajos realizados y se dio respuesta a los interrogantes en el marco de las categorías establecidas.

\section{RESULTADOS}

A partir del desarrollo metodológico se logró identificar las siguientes categorías como referentes teóricos para el desarrollo de la investigación; se hace un recuento de los conceptos y su forma de aplicación en contextos reales a 
partir de experiencias de los autores referenciados.

Ecoturismo o turismo de naturaleza. Mucho se ha dicho sobre el concepto de ecoturismo y su impacto en las dimensiones sociales, económicas y ambientales; en el estudio efectuado por Cobbinah (2015) se presenta una contextualización de los conceptos de ecoturismo a lo largo del tiempo; aunque no se puede hablar sobre un término en específico, logrando abstraer de las definiciones presentadas en el estudio se puede establecer como una participación del ser humano en un escenario geográfico, en donde lo importante es el adecuado uso de los recursos ambientales evitando el deterioro y su aniquilación en el tiempo. Pero claramente esta definición no abarca la problemática planteada desde lo teórico hacia lo práctico, donde en muchas partes del mundo solo se usa el término como estrategia de venta aunque realmente no exista una conciencia ambiental sobre el uso sustentable de los recursos.

Otros estudios hablan sobre el valor de la "actitud" frente al ecoturismo, donde las personas presentan un comportamiento de satisfacción frente al servicio ofrecido y la retribución otorgada por el mismo, dejando a un lado la sostenibilidad solo para mantener el negocio en la línea del tiempo pero dejándola como un factor necesario para obtener ganacias dentro de la oferta ecoturística (Hiu et al., 2014).

El ecoturismo ha tomado fuerza en el desarrollo de las comunidades y en la generación de ingresos económicos para las mismas. En el Foro Económico Mundial del año 2013 se señalan ingresos de alrededor de 6 mil millones de dólares al año en todo el mundo por este concepto (Lenao y Basupi, 2016; Mogrovejo et al., 2019); de ahí la importancia como estrategia de desarrollo social y económico de comunidades apartadas.

Ecoturismo comunitario. En el ámbito del ecoturismo aparece un actor valioso, las comunidades o asociaciones comunitarias que están ofreciendo servicios no formalizados pero atrayentes para las masas; ejemplo de esto se puede encontrar en las comunidades indígenas asociadas con operadores turísticos locales y nacionales (Guala y Szmulewicz, 2018), ofreciendo servicios ecoturísticos a nivel general. Sin embargo, en este tipo de ecoturismo promovido altamente por los gobiernos locales como alternativas económicas y sociales se requiere un acompañamiento y formación de los pobladores para evitar la sobreexplotación de los recursos naturales.

Experiencias como la de "Yunguilla" (Vera et al., 2017, p. 431) presentan un escenario en donde la comunidad se organizó y cambió su forma de vivir pasando de una economía "ilícita" a una economía comunitaria organizada, logrando en el turismo comunitario una forma de mejorar sus condiciones socioeconómicas y contribuyendo al mejoramiento del medio ambiente. Los habitantes se organizaron y alrededor de capacitaciones, apuestas individuales y gubernamentales, lograron sacar adelante su proyecto ecoturístico con visitas de alrededor de 3.000 personas al año.

Educación ambiental. La educación ambiental ha sido un mecanismo de los gobiernos para fomentar las transformaciones culturales con el propósito de buscar la preservación, cuidado y sustentabilidad del ambiente. Desde la Conferencia de Naciones Unidas de 1992 sobre Medio Ambiente y Desarrollo se ha hablado de educación para el desarrollo sostenible (Nasibulina, 2015), en la que se plantea una educación para fomentar el desarrollo de las comunidades y la explotación de los recursos de manera equilibrada; sin embargo, la educación ambiental ha evolucionado hacia una filosofía de vida y una forma de ver el diario vivir en donde la persona ya no es el epicentro sino, por el contrario, es parte de un sistema, el cual debe ser cuidado y preservado para que pueda seguir existiendo.

La educación ambiental busca cambiar los comportamientos de las personas, contrario a lo proambiental; en este último proceso se busca educar al individuo y la sociedad en elementos transformadores para generar conciencia, pero todavía existen muchos vacíos en la formación de las sociedades y esto se evidencia en la necesidad del ser humano de consumir cada vez mayores cantidades de energía y recursos de manera indiscriminada (Páramo, 2017). Hoy día el hombre no se agrupa para sobrevivir, sino para prosperar, y en ese proceso (Sánchez y Campos, 2018) se vuelve un depredador de su entorno con el propósito de generar una economía sostenible en el tiempo.

Desarrollo económico y social. El desarrollo económico se basa en la capacidad de las comunidades para crear oportunidades de empleo o ingresos para la población, permitiendo tener un poder adquisitivo para mejorar las condiciones sociales; sin embargo, este último no solo depende del individuo sino de los entes territoriales a través de políticas para impactar el modo de vida de sus ciudadanos; en este sentido el desarrollo económico local (Gonzáles, 2017) se puede establecer en la capacidad de las comunidades para generar fuentes de ingresos y cambiar su forma de vida.

Cuando se habla de un desarrollo social, se puede hablar de múltiples dimensiones: "rezago educativo, acceso a los 
servicios de salud, acceso a la seguridad social, calidad y espacios de la vivienda, acceso a los servicios básicos en la vivienda, acceso a la alimentación" (Ramos, 2016, p. 13). Para lograrlo, sin embargo, es necesario recordar el aspecto ambiental, sin el cual lo demás solo es temporal; el cuidado y el cambio de cultura en una sociedad buscando un equilibrio con la naturaleza logran un desarrollo sostenible. La búsqueda de soluciones para abandonar la pobreza se puede resumir como las alternativas de desarrollo social; sin embargo, es importante resaltar la calidad de vida de los ciudadanos como otro factor clave en este tema, y el hecho de que en algunas poblaciones se ha avanzado en la lucha contra la pobreza sin dejar a un lado la sustentabilidad de los ecosistemas (Vélez y Valencia, 2015).

A continuación se presenta un análisis geográfico de los casos de éxito o impactos negativos en el uso del ecoturismo implementado en el mundo globalizado como alternativa de desarrollo económico y social.

\section{México}

En México desde 1995 el gobierno firmó acuerdos para el cuidado y la protección de los recursos naturales, promoviendo el bienestar de las comunidades rurales. En este país se pasó del turismo de sol y playa hacia el "movimiento lento", el cual busca identificarse con las raíces culturales de la población y mostrar esto como atractivo turístico (Micheli, 2002).

Según Dickinson et al. (2015), "En el puerto de Celestún, la población se dedica mayormente a la pesca de mero, cazón, corvina, pámpano y pulpo; a la explotación de las salinas y en los últimos años, a la captura de pepino de mar y al ecoturismo" (p. 2). De la población existente, $25 \%$ se encuentra en la pobreza, y se ha visto en el ecoturismo una alternativa para mejorar los ingresos, pero se presentó un fenómeno con la venta del pepino de mar, el cual es comercializado de manera ilegal; esto ha afectado todo el ecosistema visitado por los turistas y constituye un ejemplo claro de un desarrollo económico generado por una cultura ilegal en donde se afecta todo el sistema. La reserva ha sobrevivido, pero requiere el apoyo gubernamental, el cual también es permeado por el fenómeno de la corrupción. Según Dickinson et al. (2015), el estudio mencionado es un ejemplo negativo de cómo se logra avanzar social y económicamente a partir del uso indiscriminado de un recurso natural. Las asociaciones de lancheros y las personas dedicadas al cuidado del manglar y demás escenarios buscan apoyo gubernamental para evitar el deterioro de una de sus fuentes principales de ingresos.

México ha desarrollado una cultura gastronómica alrededor de las tradiciones indígenas milenarias y las combina con el etno-turismo en un esfuerzo de desarrollo económico y social de las comunidades vulnerables y pobres del país. Para lograr esto ha organizado asociaciones indígenas apoyadas por el gobierno federal y los gobiernos locales para generar un renglón en la economía a partir de micro-innovaciones en las comunidades, logrando ser aceptados elementos "modernos" para mejorar la venta de servicios en el ecoturismo, siempre con el propósito de mantener la sustentabilidad del entorno, condición no negociable por los indígenas. En el estudio se mencionan las categorías "incorporación de nuevos ingredientes, incorporación de nuevas actividades, adecuación en la organización para atender a visitantes, adecuación de elementos, adecuación de servicios" (Velázquez y Gómez, 2017, p. 78). Estas micro-innovaciones, según concluye el estudio, representan una forma de adecuación de las comunidades al desarrollo.

\section{España}

La zona de estudio es una garganta semi-natural de la laguna del mar Menor, entre la cuenca principal de la laguna y el mar Mediterráneo, la cual a través de su barrera de cierre (La Manga) es la última comunicación funcional entre las cuencas (Robledano et al., 2018). En esta zona se concentran múltiples escenarios propicios para el ecoturismo, donde hay características únicas desde el punto de vista de la flora y la fauna.

En el estudio se presenta una metodología para medir los impactos socioeconómicos y ambientales de la zona de estudio; a partir de un análisis cartográfico e histórico se logró establecer el avance de los impactos positivos en lo socioeconómico relacionados con los impactos negativos en lo ambiental. Además se presentó de manera clara las diferentes zonas turísticas de influencia costera. Se analizaron las diferentes poblaciones de animales presentes en la zona y la evolución demostrada por la presencia del ser humano como actor interesado en diversión y alimentación. Se demostró la necesidad de la formulación de planes de gestión para mitigar los impactos ambientales negativos generados por el ecoturismo. Es evidente que al usar mecanismos de evaluación se pueden suplir los planes de gestión, siempre y cuando se haga una retroalimentación con la población local y esto se acompañe de educación ambiental para la preservación de los diferentes ecosistemas.

Estudios como el de Varela et al. (2018), Casaló y Escario (2018), han demostrado la importancia de la educación ambiental en el noreste de España desde las ópticas interpersonal, motivacional y educativa, analizando las variables para definir un comportamiento verde en los 
ciudadanos. El desarrollo de un meta-análisis empírico evalúa los impactos sociodemográficos y los factores psicológicos en las personas para lograr un comportamiento ecológico. Lo anterior refuerza la necesidad de tener presente la educación ambiental para lograr un desarrollo socioeconómico a lo largo del tiempo, cambiando la conciencia y cultura de las personas administradoras del territorio utilizado para el ecoturismo. Sin embrago, el estudio expone la necesidad de cambiar la forma de pensar de los ciudadanos, dada la importancia de una cultura verde para impactar en todos los ecosistemas de manera global.

\section{China}

Es la nación más poblada del mundo, en la cual el modelo económico y social gira alrededor de la producción para hacer crecer su economía. Pero a diferencia de otras naciones alrededor del mundo, ha incrementado sus paisajes verdes en los últimos años y la población ha contribuido a mejorar los diferentes escenarios de bosques, humedales y pastizales, al igual que la explotación de sus mares. China por tener escenarios naturales y una tradición milenaria, logra desarrollar de modo natural el ecoturismo; sin embargo, se ve enfrentada a los impactos negativos generados por las masas de personas presentes en estos sitios turísticos. Artículos como el de Xu et al. (2017) presentan un marco de trabajo con teoremas desde el enfoque sistémico, el cual analiza las condiciones del ecoturismo como un sistema integrado con el medio ambiente. Los elementos de este marco regulatorio de un sistema de ecoturismo son: subsistema social y cultural (participación comunitaria, distribución de beneficios, políticas y sistemas, costumbres populares, entre otras), subsistema natural (recursos naturales, paisaje, ambiente ecológico, servicios ecosistémicos, capacidad de carga), subsistema económico (comida, transporte, compras, acomodación, entretenimiento y turismo).

En el trabajo de Sun et al. (2018) se discute sobre el nivel de consumo de recursos que tiene un grupo de personas consideradas "ambientalistas", comparado con las personas que carecen de una filosofía ambientalista; los resultados sistematizados indican: los ambientalistas eligen usar elementos de eficiencia energética y apuntan a conservar los recursos, y los no ambientalistas prefieren tener electrodomésticos y vehículos tradicionales. Además, se identificó una correspondencia entre los ingresos y la educación de los encuestados. Como conclusión se menciona la necesidad del gobierno chino de acentuar los esfuerzos para cambiar la actitud de los hogares con respecto a la conservación de la energía y el uso de aparatos de eficiencia energética, lo que podría ayudar a crear comportamientos más sostenibles en la comunidad.

\section{Ecuador}

Para el fomento del desarrollo del turismo en el sur de la Amazonía, Ecuador ha experimentado con el uso de tecnologías de la información como redes sociales y aplicaciones web; esto ha permitido la sistematización de los procesos y la organización de metodologías para potenciar el turismo de ciertas zonas mediante la creación de rutas y nuevos escenarios turísticos.

La provincia de Zamora Chinchipe se encuentra en la Amazonía sur de Ecuador (Quezada et al., 2018), y fue epicentro del desarrollo de dos rutas turísticas usando software con el propósito de optimizar y registrar todos los elementos presentes para ser ofrecidos a los posibles turistas. Este intercambio y uso de tecnología permitió desarrollar nuevas regiones para lograr la generación de recursos económicos en las localidades. Sin embargo, también ayuda a la educación no formal de los habitantes en lo relacionado con el cuidado del medio y sus recursos.

\section{Implementaciones positivas}

Al revisar de manera detallada las experiencias en contextos geográficos diferentes se logró determinar los siguientes elementos en la Tabla 1 como estrategias positivas en la formulación del ecoturismo como eje de desarrollo social y económico.

Tabla. 1. Estrategias para el fomento del turismo como eje de desarrollo social y económico.

\begin{tabular}{|l|c|l|l|l|}
\hline \multicolumn{1}{|c|}{ Estrategia } & Actores involucrados & \multicolumn{1}{c|}{ Beneficiarios } & País & Referencia \\
\hline $\begin{array}{l}\text { Formular políticas } \\
\text { públicas a favor del } \\
\text { cuidado de los recursos } \\
\text { naturales. }\end{array}$ & $\begin{array}{l}\text { Estado, empresarios del } \\
\text { turismo, empresarios y } \\
\text { comunidad en general }\end{array}$ & $\begin{array}{l}\text { Comunidad en } \\
\text { general y las zonas } \\
\text { turísticas }\end{array}$ & México & (Micheli, 2002) \\
\hline $\begin{array}{l}\text { Formular planes de } \\
\text { integración de la } \\
\text { gastronomía, turismo de } \\
\text { naturaleza y Estado. }\end{array}$ & $\begin{array}{c}\text { Estado, ciudadanía, } \\
\text { empresarios del } \\
\text { turismo, restaurantes. }\end{array}$ & $\begin{array}{l}\text { Turistas, empresas } \\
\text { de turismo }\end{array}$ & México & $\begin{array}{l}\text { (Velázquez y } \\
\text { Gómez, 2017). }\end{array}$ \\
\hline
\end{tabular}




\begin{tabular}{|l|c|l|l|l|}
$\begin{array}{l}\text { Estudios de impactos } \\
\text { socioeconómicos y } \\
\text { ambientales de la zona de } \\
\text { estudio, a partir de un } \\
\text { análisis cartográfico e } \\
\text { histórico. }\end{array}$ & $\begin{array}{c}\text { Zonas turísticas, } \\
\text { operadores turísticos, } \\
\text { Estado. }\end{array}$ & $\begin{array}{l}\text { Empresarios del } \\
\text { turismo. }\end{array}$ & España & $\begin{array}{l}\text { (Robledano } \text { et al., } \\
\text { 2018). }\end{array}$ \\
\hline $\begin{array}{l}\text { Programas de educación } \\
\text { no formal en materia } \\
\text { ambiental relacionada } \\
\text { con el turismo de } \\
\text { naturaleza. }\end{array}$ & $\begin{array}{l}\text { Estado, ciudadanía, } \\
\text { empresarios del } \\
\text { turismo, empresarios y } \\
\text { comunidad en general. }\end{array}$ & $\begin{array}{l}\text { Comunidad en } \\
\text { general y las zonas } \\
\text { turísticas. }\end{array}$ & España & $\begin{array}{l}\text { (Varela } \text { et al., 2018; } \\
\text { Casaló y Escario, } \\
\text { 2018). }\end{array}$ \\
$\begin{array}{l}\text { Marco regulatorio del } \\
\text { ecoturismo visto como } \\
\text { un sistema y subsistemas. }\end{array}$ & $\begin{array}{c}\text { Estado y empresarios } \\
\text { del turismo. }\end{array}$ & $\begin{array}{l}\text { Empresarios del } \\
\text { turismo, } \\
\text { empresarios y } \\
\text { comunidad en } \\
\text { general. }\end{array}$ & China & (Xu et al., 2017) \\
\hline $\begin{array}{l}\text { Programas para fomentar } \\
\text { la cultura ambiental. }\end{array}$ & $\begin{array}{c}\text { Estado y empresarios } \\
\text { del turismo. }\end{array}$ & $\begin{array}{l}\text { Comunidad en } \\
\text { general y las zonas } \\
\text { turísticas. }\end{array}$ & China & (Sun et al., 2018) \\
\hline $\begin{array}{l}\text { Uso de las TIC para } \\
\text { promoción y cuidado de } \\
\text { las zonas turísticas. }\end{array}$ & $\begin{array}{c}\text { Zonas turísticas, } \\
\text { operadores turísticos, } \\
\text { Estado. }\end{array}$ & $\begin{array}{l}\text { Comunidad en } \\
\text { general y las zonas } \\
\text { turísticas. }\end{array}$ & Ecuador & (Quezada et al., \\
2018)
\end{tabular}

Fuente: elaboración propia basada en los autores de la columna referencia.

Cada estrategia ha sido implementada en el país relacionado y han presentado casos de éxito para la promoción del turismo de naturaleza como alternativa para el desarrollo económico y social de las comunidades nativas. Los autores mencionados de modo secuencial en la sección de países presentan el desarrollo completo de las experiencias; es pertinente aclarar que estas experiencias conllevan aspectos negativos, los cuales deben ser controlados a partir de la educación y el cambio de cultura de la población; de ahí la importancia y la necesidad de implementar acciones relacionadas con una cultura ambiental fuerte para sostener las zonas turísticas y poder generar proyectos no destructivos del medio ambiente.

\section{Implementaciones negativas}

Al analizar los estudios efectuados y ver sus resultados también se encuentran aspectos negativos producidos por el turismo natural o la creación de empresas turísticas. A continuación se hará un desglose de los aspectos negativos de los casos de éxito antes mencionados, en la Tabla 2.

Tabla. 2. Aspectos negativos en el fomento del turismo como eje de desarrollo social y económico

\begin{tabular}{|l|c|c|c|}
\hline \multicolumn{1}{|c|}{ Aspectos negativos } & Actores involucrados & País & Referencia \\
\hline Comercio ilegal de especies nativas & $\begin{array}{c}\text { Estado, comunidad en } \\
\text { general }\end{array}$ & México & (Dickinson et al., 2015) \\
\hline $\begin{array}{l}\text { Resistencia de las poblaciones } \\
\text { nativas a los cambios introducidos }\end{array}$ & $\begin{array}{c}\text { Estado, poblaciones } \\
\text { indígenas, empresarios del } \\
\text { turismo, restaurantes. }\end{array}$ & México & $\begin{array}{c}\text { (Velázquez y Gómez, } \\
\text { 2017). }\end{array}$ \\
\hline $\begin{array}{l}\text { Necesidad de la formulación de } \\
\text { planes de gestión para mitigar los } \\
\text { impactos ambientales negativos } \\
\text { generados por el ecoturismo }\end{array}$ & $\begin{array}{c}\text { Zonas turísticas, } \\
\text { operadores turísticos, } \\
\text { Estado. }\end{array}$ & España & (Robledano et al., 2018) \\
\hline
\end{tabular}


Edwin Eduardo Millán-Rojas, Verenice Sánchez-Castillo y Carlos Alberto Gómez-Cano

\begin{tabular}{|l|c|c|c|}
$\begin{array}{l}\text { Resistencia a una cultura verde por } \\
\text { parte de actores sociales y } \\
\text { comerciales }\end{array}$ & $\begin{array}{c}\text { Estado, ciudadanía, } \\
\text { empresarios del turismo, } \\
\text { empresarios y comunidad } \\
\text { en general }\end{array}$ & España & $\begin{array}{c}\text { (Varela } \text { et al., 2018), } \\
\text { (Casaló } \text { et al., 2018) }\end{array}$ \\
\hline $\begin{array}{l}\text { Resistencia de las comunidades para } \\
\text { generar cambios culturales }\end{array}$ & $\begin{array}{c}\text { Estado y empresarios del } \\
\text { turismo }\end{array}$ & China & (Xu et al., 2017) \\
\hline $\begin{array}{l}\text { Uso indiscriminado de fuentes de } \\
\text { energía tradicionales }\end{array}$ & $\begin{array}{c}\text { Estado y empresarios del } \\
\text { turismo }\end{array}$ & China & (Sun et al., 2018) \\
\hline $\begin{array}{l}\text { Carencia de acceso a las TIC por } \\
\text { parte de poblaciones rurales }\end{array}$ & $\begin{array}{c}\text { Zonas turísticas, } \\
\text { operadores turísticos, } \\
\text { Estado. }\end{array}$ & Ecuador & (Quezada et al., 2018) \\
\hline
\end{tabular}

Fuente: elaboración propia basada en los autores de la columna referencia.

La compilación de estos aspectos negativos está por fuera de los rangos normales ocasionados de manera tradicional, como la deforestación, el cambio climático, la ampliación de la frontera urbana, entre otros. Estos aspectos negativos mencionados hacen parte de las consecuencias particulares de las zonas estudiadas por los trabajos citados previamente, encontrando un patrón peculiar en los problemas generados, la carencia de una formación ambiental en las comunidades.

\section{DISCUSIÓN}

Cuando se empieza a dar respuesta a las siguientes preguntas, ¿cuáles son los vacíos conceptuales presentes en el uso del ecoturismo como alternativa de desarrollo económico y social?, ¿cómo se desarrolla el ecoturismo a lo largo de los años en las comunidades?, ¿cuál es la discusión actual sobre el ecoturismo? y ¿cuáles son los temas relevantes del ecoturismo enfocado al desarrollo económico y social de las poblaciones?, se encuentran visiones culturales diferentes, generando interés en el ecoturismo en aspectos relevantes en sus sociedades. Sin embargo, hay conceptos contradictorios en la conservación y cuidado de los recursos naturales; aunque los problemas ambientales son los mismos en puntos geográficos apartados, la influencia cultural y el interés económico por la explotación de los recursos naturales son factores que impiden a las instituciones encargadas del cuidado del medio ambiente ejecutar propuestas para dar solución a los problemas del uso excesivo de los recursos.

El ecoturismo se afianza como una alternativa para las poblaciones más pobres y olvidadas por los renglones tradicionales de la economía; sin embargo, es importante dejar claro que a la fecha de hoy no se puede dar una respuesta concluyente a las preguntas del presente estudio, dada la falta de información de todos los sitios en el mundo que han estado dedicados al ecoturismo y los nuevos sitios que están empezando a ser utilizados para este fin. Por esta razón se deja abierta la posibilidad de seguir trabajando en esta línea de investigación y dar respuesta de manera detallada a las preguntas inicialmente planteadas en la metodología del presente artículo.

\section{CONCLUSION}

Los aspectos teóricos de educación ambiental, ecoturismo y desarrollo económico y social comunitario están entrelazados de manera definitiva para lograr una estabilidad en las actividades de turismo con fines comerciales; es de señalar la necesidad de estos referentes para lograr una sostenibilidad de las zonas turísticas en el tiempo y potenciar al ciudadano como agente facilitador y mediador en el cuidado del medio y las economías locales.

Los problemas presentes en la implementación del turismo de naturaleza o ecoturismo son comunes en diferentes partes geográficas del mundo, evidenciando claramente la carencia de una educación ambiental no formal fuerte en las comunidades rurales, ocasionando explotación inadecuada de los recursos naturales y, por ende, la pérdida de un renglón económico para los diferentes países. Lo anterior ha llamado la atención de los gobiernos, los cuales buscan con políticas públicas remediar el problema del desinterés de las comunidades por cuidar sus recursos. Sin embargo, esto no ha sido suficiente, debido a aspectos de inoperancia gubernamental y esto se videncia sobremanera en países de Latinoamérica.

El ecoturismo o turismo de naturaleza representa un valioso renglón de la economía moderna; cada día los seres humanos buscan más la interacción con la naturaleza, lo cual lleva a producir ganancias momentáneas a comunidades locales, dinamizando las economías rurales y nacionales; sin embargo, es necesario lograr la creación de clúster y generar estrategias comerciales acompañadas de 
una educación ambiental para la promoción de una cultura ciudadana y comercial hacia lo sustentable y sostenible.

Los impactos positivos generados desde el ecoturismo promueven el desarrollo de renglones comerciales, logrando ganancias para todos los renglones de la economía en los países en desarrollo. Es importante tener en cuenta las estrategias implementadas en países como España y China, donde su economía no solo depende del turismo; sin embargo, este renglón ha venido situándose en un lugar importante y las experiencias adquiridas podrían servir a los países en vía de desarrollo como Colombia. Es de anotar la importancia de analizar en conjunto las experiencias negativas y positivas para crear planes de contingencia para minimizar las consecuencias, pero a la vez ver el desarrollo del turismo local como una alternativa sostenible y sustentable para las regiones apartadas de los países en desarrollo.

\section{Declaración sobre conflictos de interés}

Las ideas planteadas en este documento, así como las opiniones, comentarios y conclusiones son responsabilidad de los autores de esta y son independientes de su institución de afiliación. Así mismo, durante la realización del trabajo y la redacción de este documento no se ha incurrido en ningún conflicto de interés. El presente trabajo está enmarcado en el proyecto de investigación "Estrategias socioculturales y ambientales para el fomento del ecoturismo comunitario en el municipio de Florencia, Caquetá", desarrollado por investigadores de la Universidad de la Amazonia y financiado por el "Patrimonio autónomo Fondo Nacional de Financiamiento para la Ciencia, la Tecnología y la Innovación Francisco José de Caldas" en convenio con Colciencias, ahora Ministerio de Ciencia y Tecnología e Innovación de Colombia.

\section{REFERENCIAS BIBLIOGRÁFICAS}

Casaló, L., y Escario, J. (2018). Heterogeneity in the association between environmental attitudes and pro-environmental behavior: A multilevel regression approach. Journal of Cleaner Production, $\quad 175, \quad$ 155-163. http://doi.org/10.1016/j.jclepro.2017.11. $\underline{237}$

Cobbinah, P. B. (2015). Contextualising the meaning of ecotourism. Tourism Management Perspectives, 16, 179-189. https://doi.org/10.1016/j.tmp.2015.07.01 $\underline{5}$

Dickinson J.; Pinkus, M.; Pinkus, M., y Ramón, C. (2015). Depredación y ecoturismo: Realidades de los prestadores de servicios en la Reserva de la Biosfera Ría Celestún, Yucatán. Península, 10(1), 145-161.

http://www.scielo.org.mx/scielo.php?pid $=\mathrm{S} 1870-$

$\underline{57662015000100007 \& \text { script=sci_arttext }}$

Gonzáles, L. (2017). Desarrollo económico local: leyendas y realidades. Territorios, (1), 9-24.

https://revistas.urosario.edu.co/index.ph $\mathrm{p} /$ territorios/article/view/5605

Guala, C., y Szmulewicz, P. (2018). Evaluación de buenas prácticas en servicios de ecoturismo comunitario en la ecorregión valdiviana, Chile. Gestión Turística, (8), 9-23.

http://revistas.uach.cl/index.php/gestur/a $\underline{\text { rticle/view/3468 }}$

Hiu, Y.; Lee, W., y Chen, T. (2014). Environmentally responsible behavior in ecotourism: Antecedents and implications. Tourism management, 40, 321-329.

https://doi.org/10.1016/j.tourman.2013.0 $\underline{6.013}$

Lenao, M., y Basupi, B. (2016). Ecotourism 
Edwin Eduardo Millán-Rojas, Verenice Sánchez-Castillo y Carlos Alberto Gómez-Cano

development and female empowerment in Botswana: A review. Tourism Management Perspectives, 18, 51-58. https://doi.org/10.1016/j.tmp.2015.12.02 $\underline{1}$

Mogrovejo-Andrade, J. M.; Herrera-Martínez, S. V.; Maldonado-Niño, L. G., y GarcíaTorres, N. E. (2019). El turismo como contribución al crecimiento del PIB, para el desarrollo de la economía del país. Clío América, 13(25), 298-307. https://revistas.unimagdalena.edu.co/ind ex.php/clioamerica/article/view/3414

Micheli, J. (2002). Política ambiental en México y su dimensión regional. Región y sociedad, $\quad 14(23), \quad 129-170$. https://www.researchgate.net/publicatio $\underline{\text { n/228558469_Politica_ambiental_en_M }}$ exico_y_su_dimension_regional

Nasibulina, A. (2015). Education for sustainable development and environmental ethics. Procedia-Social and Behavioral Sciences, 214, 1077-1082. https://doi.org/10.1016/j.sbspro.2015.11. $\underline{708}$

Ospina-Arias, J. C.; Díaz-Solano, B. H., y YiRodriguez , J. de J. (2019). Desarrollo turístico desde el concepto de ciudadpuerto, caso Barranquilla, Colombia. Clío América, 13(25), 264-275. https://doi.org/10.21676/23897848.3401

Páramo, P. (2017). Pro-environmental rules: An alternative for reducing the" say-do" gap in environmental education. Suma

$\begin{array}{lll}\text { Psicológica, } & \text { 24(1), }\end{array}$ http://www.scielo.org.co/scielo.php?scri $\mathrm{pt}=\mathrm{sci} \_$arttext\&pid=S0121$\underline{43812017000100042}$

Quezada-Sarmiento, P.; Suasnavas-Rodriguez, M.; Chango-Canaveral, P.; GonzagaVallejo, C.; Enciso, L., y CalderónCordova, C. (2018). Use of social networks and web application to design and promote the ecotourism route in the Southern Amazon of Ecuador. In 2018 13th Iberian Conference on Information Systems and Technologies (CISTI) (pp. 1-7). Quito: IEEE. http://10.23919/CISTI.2018.8399399

Ramos, J. M. L. (2016). Medición multidimensional de la pobreza: estado de la cuestión y aplicación al ODS-1. Revista Internacional de Cooperación y Desarrollo, 3(1), 4-34. https://doi.org/10.21500/23825014.2540 Robledano, F.; Esteve, M.; Calvo, J.; MartínezPaz, J.; Farinós, P.; Carreño, M., y Zamora, A. (2018). Multi-criteria assessment of a proposed ecotourism, environmental education and research infrastructure in a unique lagoon ecosystem: The Encañizadas del Mar Menor (Murcia, SE Spain). Journal for Nature Conservation, 43, 201-210. https://doi.org/10.1016/j.jnc.2017.10.00 7 
ECOTURISMO IMPLEMENTADO EN EL MUNDO GLOBALIZADO COMO ALTERNATIVA DE DESARROLLO ECONÓMICO Y SOCIAL

Sánchez-Bayon, A., y Campos-García-deecoturismo indígena de México. Revista Quevedo, G. (2018). Plan de acción Internacional, $\quad$ 10(3), 73-80. frente al consumismo global de la Nueva https://papers.ssrn.com/sol3/papers.cfm? Economía: Revelaciones sobre abstract_id=3040189 economía, empresa y consumo del s. XXI. Revista Empresa y Humanismo, $X X I(1)$ 69-93.

http://dx.doi.org/10.15581/015.XXI.1.69 $\underline{-93}$

Silva-Guerra, H. (2017). La innovación y educación: variables claves para la competitividad de las empresas. Clío América, 11(21). http://dx.doi.org/10.21676/23897848.20 $\underline{79}$

Sun, W.; Zhang, X.; Li, H.; Wu, J., y Zheng, S. (2018). Environmental ideology and household energy conservation in Beijing. Journal of Cleaner Production, 195 , 1600-1608.

https://doi.org/10.1016/j.jclepro.2017.07 .065

Varela-Candamio, L.; Novo-Corti, I., y GarcíaÁlvarez, M. (2018). The importance of environmental education in the determinants of green behavior: A metaanalysis approach. Journal of Cleaner Production, 170, 1565-1578. https://doi.org/10.1016/j.jclepro.2017.09 .214

Velázquez-Sánchez, R., y Gómez-Velázquez, J. (2017). Innovación-adecuación en el
Vélez, P., y Valencia, P. (2015). El crecimiento empresarial, una herramienta fundamental para la reducción de la pobreza. Clío América, 9(17), 77-84. http://dx.doi.org/10.21676/23897848.14 $\underline{83}$

Vera, L. H.; Paladines, G. V., y Velasco, J. E. (2017). Gestión del turismo comunitario como alternativa de desarrollo local. Comunidad Yunguilla. Quito. Revista Publicando, 4(11. (2)), 427-441. https://revistapublicando.org/revista/ind ex.php/crv/article/view/571/pdf_394

Xu, S.; Mingzhu, L.; Bu, N., y Pan, S. (2017). Regulatory frameworks for ecotourism: An application of total relationship flow management theorems. Tourism Management, $\quad 61, \quad 321-330$. https://doi.org/10.1016/j.tourman.2017.0 $\underline{2.012}$

Zamora-Torres, A., y Riveros-Figueroa, E. (2016). Estudio de la región Michoacana de Zamora como polo de competitividad internacional agrícola. Clío América, 10(20),

139-159.

http://dx.doi.org/10.21676/23897848.18

$\underline{73}$ 